\title{
Distributed Switching Control to Achieve Resilience to Deep Fades in Leader-Follower Nonholonomic Systems
}

\author{
Bin $\mathrm{Hu}$ \\ Department of Electrical Engineering \\ University of Notre Dame \\ Notre Dame, Indiana \\ bhu2@nd.edu
}

\author{
Michael D. Lemmon \\ Department of Electrical Engineering \\ University of Notre Dame \\ Notre Dame, Indiana \\ lemmon@nd.edu
}

\begin{abstract}
Leader-follower formation control is a widely used distributed control strategy that often needs systems to exchange information over a wireless radio communication network to coordinate their formations. These wireless networks are subject to deep fades, where a severe drop in the quality of the communication link occurs. Such deep fades may significantly impact the formation's performance and stability, and cause unexpected safety problems. In many applications, however, the variation in channel state is a function of the system's kinematic states. This suggests that one can use channel state information as a feedback signal to recover the performance loss caused by a deep fade. This paper derives sufficient conditions to assure almost-sure practical stability of a leader-follower nonholonomic system in the presence of deep fades. These conditions relate the channel state to the system's convergence rate. This paper uses this fact to switch the controller. Simulation results are used to illustrate the main findings in the paper.
\end{abstract}

\section{Categories and Subject Descriptors}

B.1.0 [Control Structures and Microprogramming]: General; H.1.1 [Models and Principles]: Systems and Information Theory-General Systems Theory

\section{Keywords}

Distributed switching control, Deep fading, Resilience, Channel state information, Almost sure practical stability

\section{INTRODUCTION}

In the past decade, formation control has found extensive applications in industry and academia $[2,12,9,11,5]$. In formation control, the agents coordinate with each other to form and maintain a specified formation. The coordination is often conducted distributedly over a wireless radio communication network. It is well known that such communication networks are subject to deep fading, which causes a severe drop in the network's quality-ofservice (QoS). These deep fades negatively impact the formation's

Permission to make digital or hard copies of all or part of this work for personal or classroom use is granted without fee provided that copies are not made or distributed for profit or commercial advantage and that copies bear this notice and the full citation on the first page. To copy otherwise, to republish, to post on servers or to redistribute to lists, requires prior specific permission and/or a fee.

Copyright 20XX ACM X-XXXXX-XX-X/XX/XX ...\$15.00. performance and stability by interfering with the coordination between agents. The loss of coordination may cause serious safety issues in applications like smart transportation system [17], unmanned aerial vehicles system[14] and underwater autonomous vehicles[13]. These issues can be addressed by developing a resilient control system that detect such deep fades and adaptively reconfigures its controller to maintain a minimum performance level.

Channel fading is often characterized in terms of channel gain [16]. Channel gain represents the signal strength ratio of receiving signal over transmission signal. It is usually modeled as an independent and identical distributed (i.i.d) random process with Rayleigh or Rician distribution. This model is inadequate in two aspects. First, the fading process exhibits memory which is better modeled as a Markov random process with two states [18]. Second, the i.i.d. channel model ignores the impact that the formation's kinematic states have on the channel. Vehicle-to-Vehicle (V2V)[4] systems provide an example in which the velocity and relative distance of the vehicles significantly affect the channel state. Moreover, for those wireless communication systems using directional antennae $[20,1]$, changes in the relative vehicle orientation could also lead to a deep fade.

The loss of information caused by deep fades negatively limits the performance that can be achieved by the control system. Prior work $[19,15,6]$ characterized the minimum stabilizing bit rate for linear time-invariant system assuming constant channel gain. As noted above, the assumption on constant channel gain is overly simplistic for fading channels. An initial attempt to study the impact of the time-varying channel gains on mean square stability appeared in [10]. This work, however, assumed the channel gain was functionally independent from the physical system's dynamics. In [7], the authors examined a more realistic fading channel model in which the channel is exponentially bursty and is dependent on the norm of the physical system's states. This paper extends the prior work in [7] to a two-dimensional leader-follower formation control problem.

Another unique aspect of this paper is the use of almost-sure practical stability to characterize system safety. By "safety", we mean that the probability of a system's deviation from a bounded set asymptotically goes to zero as time gets large. The asymptotic nature of such probability bounds is captured in almost-sure stability concepts. Much of the prior work has instead focused on mean-square stability (MSS) which do not provide strong safety guarantees in the sense mentioned above. The focus on almostsure characterizations of safety is therefore another unique feature of this paper.

Leader-follower formations are useful for their simplicity and scalability. This paper studies the leader-follower control scheme for nonholonomic systems using directional antennae to access the 
wireless communication network. Assuming an exponentially bursty channel model, this paper derives conditions that are sufficient for the system to have almost-sure practical stability [8]. The paper uses this characterization to propose adaptive control schemes that switch feedback controllers in response to changes in channel state. The simulation results demonstrate the merits of the proposed method.

\section{MATHEMATICAL PRELIMINARIES}

Let $\mathbb{Z}$ and $\mathbb{R}$ denote the set of integers and real numbers, respectively. Let $\mathbb{Z}_{+}$and $\mathbb{R}_{+}$denote the set of positive integers and nonnegative real numbers, respectively. Let $\mathbb{R}^{n}$ denote the $n$-dimensional Euclidean vector space. The $\infty$-norm on the vector $x \in \mathbb{R}^{n}$ is $|x|=$ $\max \left|x_{i}\right|: 1 \leq i \leq n$, and the corresponding induced matrix norm is $\|A\|=\max _{1 \leq i \leq n} \sum_{j=1}^{n}\left|A_{i}^{j}\right|$. Given a vector $x \in \mathbb{R}^{n}$, we let $x_{i} \in \mathbb{R}$ for $i=1,2, \ldots, n$ denote the $i$ th element of vector $x$. We let $f(\cdot)$ : $\mathbb{R} \rightarrow \mathbb{R}^{n}$ denote a function mapping the real line onto vectors in $\mathbb{R}^{n}$.

Let $f(t) \in \mathbb{R}^{n}$ denote the value that function $f$ takes at time $t \in \mathbb{R}$. Let $\left\{\tau_{k}\right\}_{k=0}^{\infty}$ denote a strictly monotone increasing sequence with $\tau_{k} \in \mathbb{R}_{+}$for all $k \in \mathbb{Z}_{+}$and $\tau_{k}<\tau_{k+1}$. Then, $f\left(\tau_{k}\right)$ denotes the value of function $f$ at time $\tau_{k}$. For simplicity, we let $f(k)$ denote $f\left(\tau_{k}\right)$ if its meaning is clear in the context. The left-hand limit at $\tau_{k} \in \mathbb{R}$ of a function $f(\cdot): \mathbb{R} \rightarrow \mathbb{R}^{n}$ is denoted by $f\left(k^{-}\right)$. Similarly, the right-hand limit of the function $f(k)$ is denoted by $f\left(k^{+}\right)$.

Consider a continuous-time random process $\left\{x(t) \in \mathbb{R}^{n}: t \in \mathbb{R}_{+}\right\}$ whose sample paths are right-continuous and satisfy the following differential equation,

$$
\dot{x}(t)=f(x(t), u(t), w(t), d(t))
$$

where $f(0,0,0,0)=0, u(\cdot): \mathbb{R}_{+} \rightarrow \mathbb{R}^{m}$ is a control input, $d(t)$ is an external $\mathscr{L}_{\infty}$ disturbance with $|d(t)|_{\mathscr{L}_{\infty}}=D$ and $w(t)$ is a jump process

$$
w(t)=\sum_{\ell=1}^{\infty} w_{\ell} \delta\left(t-\tau_{\ell}\right)
$$

in which $\left\{w_{\ell}, \ell \in \mathbb{Z}_{+}\right\}$is a Markov process describing the $\ell$ th jump's size at jump instants $\left\{\tau_{\ell}\right\}_{\ell=1}^{\infty}$. The expectation of this stochastic process at time $t$ will be denoted as $\mathbb{E}(x(t))$.

The system in equations (1-2) is said to be almost-surely practical stable if for all $\varepsilon>0$ and $\rho>0$, there exists $T>0$ and $(\delta, \lambda)$ with $\lambda>\delta>0$ such that if $|x(0)| \leq \delta$, then

$$
\operatorname{Pr}\left\{\sup _{t \geq T}|x(t)|-\lambda \geq \varepsilon\right\} \leq \rho
$$

Given a dynamical system (1-2) and a set, $\Omega \subset \mathbb{R}^{n}$, of safe states one says this system is almost-surely safe if the system is almostsurely practically stable with respect to the set of safe states, $\Omega$.

\section{SYSTEM DESCRIPTION}

\subsection{System Model}

Figure 1 shows a string formation of $N$ mobile robots. For each mobile robot, we consider the following kinematic model,

$$
\dot{x}_{i}=v_{i} \cos \left(\theta_{i}\right), \dot{y}_{i}=v_{i} \sin \left(\theta_{i}\right), \dot{\theta}_{i}=\omega_{i}, i=0,1, \ldots, N-1
$$

where $\left(x_{i}(t), y_{i}(t)\right)$ denotes the vehicle $i$ 's position at time $t \in \mathbb{R}_{+}$, $\theta_{i}(t)$ is the orientation of the vehicle relative to the $x$ axis at time $t$. $v_{i}$ and $\omega_{i}$ are the vehicle's speed and angular velocity that represent the control input.

As shown in Figure 1, the cascaded formation with $N$ mobile robots consists of $N-1$ leader-follower pairs. In each leader-follower

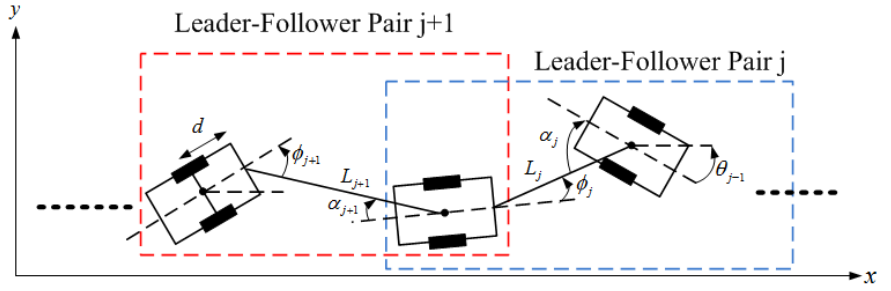

Figure 1: A cascaded formation of nonholonomic vehicular system

pair $j$, we assume that the leader can directly measure its relative bearing angle $\alpha_{j}$ to the follower. Similarly, the follower can measure its bearing angle $\phi_{j}$ to the leader. Both of the vehicles are able to measure the relative distance $L_{j}$. What is not directly known to the follower is the relative bearing angle $\alpha_{j}$. In this paper, we consider the case when information about leader's bearing angle $\alpha_{j}$ is transmitted over a wireless channel. The channel is accessed through a directional antenna whose radiation pattern is shown in Figure 2.

The control objective of the cascaded formation is to have the follower in each leader-follower pair to regulate its speed and angular velocity to achieve and maintain a desired distance and bearing angle. Let $L_{d_{j}}$ and $\alpha_{d_{j}}$ denote the desired inter-vehicle distance and relative bearing angle, respectively, in the $j$ th leader-follower pair. It will therefore be convenient to characterize the time rate of change of the relative distance $L_{j}$ and leader's relative bearing angle $\alpha_{j}$ as follows [5]

$$
\begin{aligned}
& \dot{L}_{j}=v_{j-1} \cos \alpha_{j}-v_{j} \cos \phi_{j}-d \omega_{j} \sin \phi_{j} \\
& \dot{\alpha}_{j}=\frac{1}{L_{j}}\left(-v_{j-1} \sin \alpha_{j}-v_{2} \sin \phi_{j}+d \omega_{j} \cos \phi_{j}\right)+\omega_{j-1}
\end{aligned}
$$

where $d$ is the distance from the vehicle's center to its front.

\subsection{Information Structure}

As discussed in the previous section, the leader's bearing angle $\alpha_{j}$ in each leader-follower pair must be transmitted to the follower over a wireless channel. In this regard, the information about $\alpha_{j}$ that is available to the follower is limited by the following two constraints,

- The state measurement $\alpha_{j}(t)$ is only taken at a sequence of time instants $\left\{\tau_{k}\right\}_{k=0}^{\infty}$ that satisfies $\tau_{k}<\tau_{k+1}, k=1,2, \ldots, \infty$.

- The sampled data $\alpha_{j}\left(\tau_{k}\right)$ is quantized with a finite number of bits $\bar{R}_{j}$, and is transmitted over an unreliable wireless channel with only first $R_{j}(k)$ bits $\left(R_{j}(k) \leq \bar{R}_{j}\right)$ received at the follower.

At $k$ th sampling time instant, the triple $\left\{\hat{\alpha}_{j}\left(k^{-}\right), U_{j}(k), c_{j}(k)\right\}$ characterizes the information structure of the leader's bearing angle $\alpha_{j}\left(\tau_{k}\right)$ at the leader side. Assume that the measurement $\alpha_{j}\left(\tau_{k}\right)$ lies in an interval $\left[-U_{j}(k)+\hat{\alpha}_{j}\left(k^{-}\right), U_{j}(k)+\hat{\alpha}_{j}\left(k^{-}\right)\right]$with $\hat{\alpha}_{j}\left(k^{-}\right)$ representing the "center" of the interval and $U_{j}(k)$ representing the length of the interval. The codeword $c_{j}(k)=\left\{b_{j l}(k)\right\}_{l=1}^{\bar{R}_{j}}$ consists of bits $b_{j l}(k) \in\{-1,1\}$, and is constructed by truncating the first $\bar{R}_{j}$ bits of the following infinity bit sequence

$B_{j}(k):=\left\{\left\{b_{j l}(k)\right\}_{l=1}^{\infty} \in\{-1,1\}^{\infty} \mid \alpha_{j}\left(\tau_{k}\right)=\hat{\alpha}_{j}\left(k^{-}\right)+U_{j}(k) \sum_{l=1}^{\infty} \frac{1}{2^{j}} b_{j l}(k)\right\}$

This corresponds to a uniform quantization of the sampled state within the interval $\left[-U_{j}(k)+\hat{\alpha}_{j}\left(k^{-}\right), U_{j}(k)+\hat{\alpha}_{j}\left(k^{-}\right)\right]$with $\bar{R}_{j}$ number of bits. 
We assume that the follower only successfully receives the first $R_{j}(k)$ bits in the codeword $c_{j}(k)$. The information structure at the follower side is another triple $\left\{\hat{\alpha}_{j}(k), U_{j}(k), \hat{c}_{j}(k)\right\}$ with $\hat{c}_{j}(k)=$ $\left\{b_{j l}\right\}_{l=1}^{R_{j}(k)}$ and $\hat{\alpha}_{j}(k)$ being constructed as follows

$$
\hat{\alpha}_{j}(k)=\hat{\alpha}_{j}\left(k^{-}\right)+U_{j}(k) \sum_{l=1}^{R_{j}(k)} \frac{1}{2^{j}} b_{j l}(k) .
$$

$\hat{\alpha}_{j}(k)$ is an estimate of the leader's bearing angle $\alpha_{j}(k)$ at time instant $\tau_{k}$.

In order to reconstruct the estimate $\hat{\alpha}_{j}(k)$, it is necessary to synchronize the leader and follower in the sense that they have the same information structure. We assume a noiseless feedback channel, with each successfully received bit being acknowledged to the leader. This allows one to ensure that the information structures are synchronized between the leader and follower.

The follower then uses the estimated bearing angle $\hat{\alpha}_{j}(k)$, and the measured inter-vehicle distance $L_{j}$, to select its speed, $v_{j}$, and angular velocity $\omega_{j}$ to achieve the control objective.

\subsection{Wireless Channel}

As shown in Figure 2, the leading vehicle in each pair uses a directional antenna to access the wireless channel. We assume the channels are free of interference from other leader-follower pairs, but the channel does exhibit deep fading. Deep faces occur when the channel gain drops below a threshold and stays below that threshold level for a random interval of time. Such fades are often modeled using two-state Markov chains[18].

We adopt an exponentially bounded burstiness (EBB) characterization of the fading channel. In particular, let $h(\cdot, \cdot)$ and $\gamma(\cdot, \cdot)$ denote continuous, positive and monotone decreasing functions from $\mathbb{R}_{+} \times \mathbb{R}_{+}$to $\mathbb{R}_{+}$. Assume the probability of successfully decoding $R_{j}(k)$ bits at each sampling time $\tau_{k}$ satisfies

$$
\begin{gathered}
\operatorname{Pr}\left\{R_{j}(k) \leq h\left(\left|\alpha_{j}\left(\tau_{k}\right)\right|,\left|L_{j}\left(\tau_{k}\right)\right|\right)-\sigma\right\} \leq e^{-\gamma\left(\left|\alpha_{j}\left(\tau_{k}\right)\right|,\left|L_{j}\left(\tau_{k}\right)\right|\right) \sigma} \\
\text { for }\left|\alpha_{j}\left(\tau_{k}\right)\right| \leq \pi / 2 \text { and } \sigma \in\left[0, h\left(\left|\alpha_{j}\left(\tau_{k}\right)\right|, \mid L_{j}\left(\tau_{k}\right)\right) \mid\right] \text { with } \\
\operatorname{Pr}\left\{R_{j}(k)=0\right\}=1
\end{gathered}
$$

for $\left|\alpha_{j}\left(\tau_{k}\right)\right|>\pi / 2, \forall k \in \mathbb{Z}_{+}$. We say such channels exhibit exponentially bounded burstiness (EBB). EBB characterizations can be used to describe a wide range of Markov channel models that include traditional i.i.d models as well as two-state Markov chain models. The analysis methods in this paper apply to a wide range of realistic channel conditions.

The equations (6) and (7) characterize the fact that if the follower vehicle is out of the antenna's radiation scope, i.e. $\left|\alpha_{j}\left(\tau_{k}\right)\right|>\pi / 2$, then the communication link between the vehicles is broken. If the vehicle is within the scope, i.e. $\left|\alpha_{j}\left(\tau_{k}\right)\right| \leq \pi / 2$, the probability of having a low bit rate is exponentially bounded.

As shown in Figure 2, the function $h\left(\left|\alpha_{j}\right|,\left|L_{j}\right|\right)$ in EBB model may be seen as a threshold characterizing the low bit rate region as a function of current formation's state. The exponent associated with exponential decrease is represented by a similar function $\gamma\left(\left|\alpha_{j}\right|,\left|L_{j}\right|\right)$. The two functions play different roles in the EBB model. Function $h\left(\left|\alpha_{j}\right|,\left|L_{j}\right|\right)$ characterizes the fact that as the absolute value of the formation's state $L$ and $\alpha$ increase, the low bit rate threshold shrinks and moves toward the origin. Such activity can be induced due to path loss that is widely considered in the wireless communication community. On the other hand, the function $\gamma\left(\left|\alpha_{j}\right|,\left|L_{j}\right|\right)$ in the exponential bound models the fact that the likelihood of exhibiting a low bit rate increases as the formation state is away from the origin.
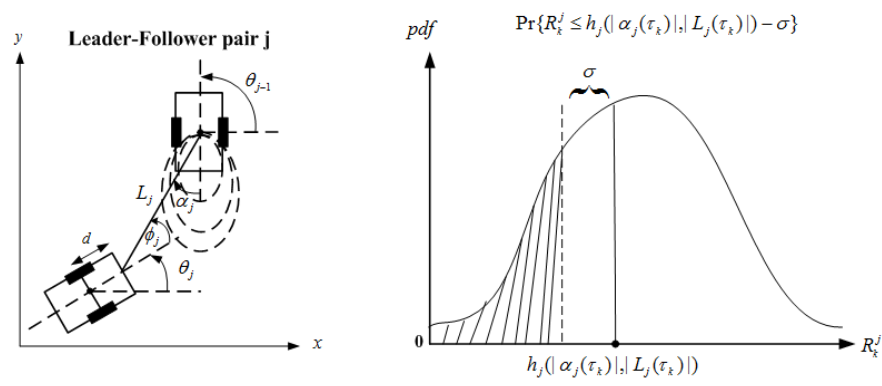

Figure 2: Exponential Bounded Burstiness (EBB) Model for directional wireless channel

What should be apparent from the EBB model is that we are explicitly accounting for the relationship between channel state $\left(R_{j}(k)\right)$ and formation configuration. A major goal of this paper is to exploit that relationship in deciding how to switch between different controllers to assure almost sure performance.

\subsection{Distributed Switching Control}

In this paper, the control objective is to steer the cascaded vehicular system shown in Figure 1 to a sequence of desired distances $\left\{L_{d_{j}}\right\}_{j=1}^{N-1}$ and bearing angles $\left\{\alpha_{d_{j}}\right\}_{j=1}^{N-1}$ in a distributed fashion, and then maintain around those set-points.

At each time instant $\left\{\tau_{k}\right\}_{k=0}^{\infty}$, the follower of each leader-follower pair switches among a group of controller gains to regulate its velocity and angular velocity to achieve the control objective. Let $K(k):=\left\{K_{\alpha_{j}}(k), K_{L_{j}}(k)\right\}$ denote the controller gain pair used for leader-follower pair $j$ at time instant $\tau_{k}$. These controller gains are selected from one pair of a collection of values $\mathscr{K}_{j}=\left\{K_{j_{1}}, K_{j_{2}}, \ldots, K_{j_{M}}\right\}$. Recall that the dynamics of formation configuration is equation (4), we use standard input to state feedback linearization to generate the control input

$$
\left[\begin{array}{c}
v_{j} \\
\omega_{j}
\end{array}\right]=\left[\begin{array}{rr}
-\cos \phi_{j} & -L_{j} \sin \phi_{j} \\
-\frac{\sin \phi_{j}}{d} & \frac{L_{j}}{d} \cos \phi_{j}
\end{array}\right]\left[\begin{array}{c}
K_{L_{j}}(k)\left(L_{d_{j}}-L_{j}\right) \\
K_{\alpha_{j}}(k)\left(\alpha_{d_{j}}-\hat{\alpha_{j}}\right)
\end{array}\right]
$$

over the time interval $\left[\tau_{k}, \tau_{k+1}\right)$. The variable $\hat{\alpha}_{j}(t)$ is a continuous function over $\left[\tau_{k}, \tau_{k+1}\right)$, and satisfies the following initial value problem,

$$
\dot{\hat{\alpha}}_{j}=K_{\alpha_{j}}(k)\left(\alpha_{d_{j}}-\hat{\alpha}_{j}\right), \hat{\alpha}_{j}\left(\tau_{k}\right)=\hat{\alpha}_{j}(k)
$$

where the estimate $\hat{\alpha}_{j}(k)$ is obtained from equation (5). With this control, the inter-vehicle distance $L_{j}$ and bearing angle $\alpha_{j}$ satisfy the following differential equations over $\left[\tau_{k}, \tau_{k+1}\right)$,

$$
\left[\begin{array}{c}
\dot{L}_{j} \\
\dot{\alpha}_{j}
\end{array}\right]=\left[\begin{array}{cc}
\cos \alpha_{j} & 0 \\
\frac{-\sin \alpha_{j}}{L_{j}} & 1
\end{array}\right]\left[\begin{array}{c}
v_{j-1} \\
\omega_{j-1}
\end{array}\right]+\left[\begin{array}{c}
K_{L_{j}}(k)\left(L_{d_{j}}-L_{j}\right) \\
K_{\alpha_{j}}(k)\left(\alpha_{d_{j}}-\hat{\alpha}_{j}\right)
\end{array}\right]
$$

for all $k=1,2, \ldots, \infty$.

The equations (8-9) represent the closed-loop system for the leaderfollower pair $j$ and can be viewed as an example of a jump nonlinear system given in equations (1-2). The $\mathscr{L}_{\infty}$ disturbance in the $j$ th leader-follower system is $\left[v_{j-1}, \omega_{j-1}\right]$. The estimate of the bearing angle $\hat{\alpha}_{j}$ forms a jump process with jumps occurring at discrete time instants $\left\{\tau_{k}\right\}_{k=1}^{\infty}$. As shown in equation (5), the magnitude of the jump at each time instant is stochastically governed by the length of the uncertainty interval $U_{j}(k)$ and the number of received bits $R_{j}(k)$. Such jump process significantly impacts the formation performance of the cascaded system by pushing the formation state away from the equilibrium, which in turn leads to deep fades with 
a high probability. In the next section, we will show how to reconfigure the local controller gain in response to the changes of $U_{j}(k)$ and $R_{j}(k)$ such that almost sure performance is assured.

It is apparent from Figure 1 that vehicle $j$ for $j=1,2, \ldots, N-2$ plays a leader in leader-follower pair $j+1$ as well as a follower in leader-follower pair $j$. In this regard, vehicle $j$ could observe the full state $\alpha_{j+1}$ of the leader-follower subsystem $j+1$ because it serves the leadership in that system. By observing the behavior of the following vehicle, vehicle $j$ for $j=1,2, \ldots, N-1$ can adjust its controller gain to overcome large overshoots in the following system. Such cooperative control strategy lessens the amplification on the disturbance from the upper leader-follower systems to the lower systems.

\section{MAIN RESULTS}

This paper's main results consist of two parts regarding to the behavior of inter-vehicle distance $L_{j}$ and bearing angle $\alpha_{j}$ for each leader-follower pair. The first part of the results provide a sufficient condition under which the inter-vehicle distance $L_{j}$ for $j=$ $1,2, \ldots, N-1$ is convergent to a compact invariant set. The second part of the results derive sufficient conditions for the almost sure practical stability for the bearing angle $\alpha_{j}, j=1,2, \ldots, N-1$.

In the main results, we use the fact that the leader's action in each leader-follower pair can be constrained as a function of the following system's state to assure the stability for the whole leaderfollower system. Proposition 4.1 provides an explicit characterization of the bound on the leader's action, as well as a distributed way to achieve that bound. Using the results from Proposition 4.1, one can easily prove the first main result in this paper (Lemma 4.4), i.e. the convergence of inter-vehicle distance since the distance is measurable to both leader and follower. The more challenging and interesting part of the results is to guarantee the almost sure practical stability for the bearing angle $\alpha_{j}$, which is presented in section 4.2.

The following Proposition is provided to assure the control input from upper leader-follower subsystem is bounded as a function of state estimates of the bottom system. The proof is provided in Appendix.

PROPOSITION 4.1. Consider the closed-loop system in equations (8-9), let $0<d \leq 1$, if there exists a sequence of controller gains $\left\{K_{j}(k)\right\}_{k=0}^{\infty}, K_{j}(k)=\left\{K_{L_{j}}(k), K_{\alpha_{j}}(k)\right\} \in \mathscr{K}_{j}$ such that for given bounded increasing positive functions $W_{j}(\cdot): \mathbb{R}_{+} \rightarrow \mathbb{R}_{>0}$ with $\sup _{s} W_{j}(s)=W_{j}^{*}, j=1,2, \ldots, N-1$, the following inequality holds for all $k=0,1, \ldots, \infty$

$$
\max \left\{K_{L_{j}}(k)\left(M_{L_{j}}(k)+L_{d_{j}}\right), K_{\alpha_{j}}(k)\left|\tilde{\alpha}_{j}(k)\right|\right\} \leq \frac{W_{j}\left(\left|\tilde{\alpha}_{j+1}(k)\right|\right)}{\left(1+M_{L_{j}}(k)\right)}
$$

Where

$$
\begin{aligned}
M_{L_{j}}(k) & =\max \left\{\bar{L}_{j}\left(\tau_{k}\right), \bar{L}_{j}\left(\tau_{k+1}\right)\right\} \\
\bar{L}_{j}(t) & =\left(L_{d_{j}}+\frac{W_{j-1}\left(\left|\alpha_{j}(k)\right|\right)}{K_{L_{j}}(k)}\right)\left(1-e^{-K_{L_{j}}(k)\left(t-\tau_{k}\right)}\right) \\
& +L_{j}(k) e^{-K_{L_{j}}(k)\left(t-\tau_{k}\right)} \\
\tilde{\alpha}_{j}(k) & =\alpha_{d_{j}}-\hat{\alpha}_{j}(k)
\end{aligned}
$$

then

$$
\left|\left[\begin{array}{c}
v_{j}(t) \\
\omega_{j}(t)
\end{array}\right]\right|_{\infty} \leq W_{j}\left(\left|\tilde{\alpha}_{j+1}(k)\right|\right), t \in\left[\tau_{k}, \tau_{k+1}\right)
$$

Because of inequality (11), each leader-follower subsystem $j$ in equation (9) can bound the external disturbance $\left[v_{j-1}, \omega_{j-1}\right]$ by observing its local state estimate $\tilde{\alpha}_{j}$ at each time instatnt $\tau_{k}$. Meanwhile, the subsystem $j-1$ can select its controller gain so that the control input $\left[v_{j-1}, \omega_{j-1}\right]$ satisfies the bound in inequality (11) because the estimate of bearing angle $\tilde{\alpha}_{j}$ is always available to subsystem $j-1$. Such property provides a basis to design a distributed and cooperative switching law to assure the stability for the whole formation system.

REMARK 4.2. Functions $W_{j}(\cdot)$ are upper bounds on the control inputs of upper leader-follower system and the values of $W_{j}(\cdot)$ at each time instant $\tau_{k}$ can also be seen as feedback signals from the bottom system. Such feedback signals directly constrain the magnitude of control input from upper system, so that the disturbances are not amplified from upper system to bottom system.

REMARK 4.3. The inequality (10) could be viewed as a switching rule for the leader-follower pair $j$ to react to the changes on system $j+1$ 's bearing angle. The switching rule applied over each time interval $\left[\tau_{k}, \tau_{k+1}\right)$ is feasible because it is only based on the information that is available at time $\tau_{k}$. Since the function $W_{j}$ is bounded and positive, we can always find sufficiently small controller gains such that the inequality (10) holds.

\subsection{Convergence of Inter-vehicle Distance $L_{j}$}

In this section, we present the first main result of this paper involving the convergence of inter-vehicle distance. The following lemma provides a sufficient condition on the controller gain $K_{L_{j}}$, under which one can show $L_{j}(t)$ converges at an exponential rate to an invariant set $\Omega_{\mathrm{inv}, \mathrm{j}}$ centered at the desired inter-vehicle distance $L_{d_{j}}$, for $j=1,2, \ldots, N-1$.

LEMMA 4.4. Let the hypothesis of proposition 4.1 hold, consider the system (8-9) with the selected controller gain $\left\{K_{L_{j}}, K_{\alpha_{j}}\right\} \in$ $\mathscr{K}_{j}$. If $K_{L_{j}}>\frac{W_{j}^{*}}{\delta\left(L_{d_{j}}-d\right)}$ and $L_{j}(0)>d$, then for any sample path, $L_{j}(t) \geq d$ for all $t \in \mathbb{R}_{+}$and there exists a finite time $\bar{T}>0$ such that $L_{j}(t)$ enters and remains in the set

$$
\Omega_{\mathrm{inv}, \mathrm{j}} \equiv\left\{L_{j} \in \mathbb{R}_{+}|| L_{j}-L_{d_{j}} \mid \leq \frac{W_{j}^{*}}{\delta K_{L_{j}}}\right\}
$$

for all $t \geq \bar{T}$ and any $\delta \in(0,1]$.

Proof. Consider the function $V\left(L_{j}\right)=\frac{1}{2}\left(L_{j}-L_{d_{j}}\right)^{2}$ and closedloop state equation (9). Taking the directional derivative of $V$, one obtains

$$
\begin{aligned}
\dot{V}\left(L_{j}\right) & =-K_{L_{j}}\left(L_{j}-L_{d_{j}}\right)^{2}+\left(L_{j}-L_{d_{j}}\right) \cdot v_{j-1} \cos \alpha_{j} \\
& \leq-K_{L_{j}}(1-\delta)\left(L_{j}-L_{d_{j}}\right)^{2}-\delta \cdot K_{L_{j}}\left(L_{j}-L_{d_{j}}\right)^{2}+\left|L-L_{d}\right| W_{j}^{*}
\end{aligned}
$$

for any $\delta \in(0,1]$. The last inequality holds because of proposition 4.1. When $\left|L_{j}-L_{d_{j}}\right| \geq \frac{W_{j}^{*}}{\delta K_{L_{j}}}$, the following dissipative inequality holds,

$$
\begin{aligned}
\dot{V}\left(L_{j}\right) & \leq-K_{L_{j}}(1-\delta)\left(L_{j}-L_{d_{j}}\right)^{2} \\
& =-2 K_{L_{j}}(1-\delta) V\left(L_{j}\right)
\end{aligned}
$$

This is sufficient to imply that $V\left(L_{j}(t)\right)$ is an exponentially decreasing function of time that enters the set $\Omega_{\text {inv,j }}$ in finite time. $L_{j}(t)>d$ for all time since all $L_{j}$ in $\Omega_{\mathrm{inv}, \mathrm{j}}$ satisfy

$$
L_{j} \geq-\frac{W_{j}^{*}}{\delta K_{L_{j}}}+L_{d_{j}}>d
$$


REMARK 4.5. Note that $d$ is the distance from the center of the vehicle to the front of the vehicle. As shown in Figure $1, L_{j}(t)>d$ simply means that the two vehicles do not collide with each other.

\subsection{Almost Sure Practical Stability for Bear- ing Angle $\alpha_{j}$}

In this section, we provide the second main result of this paper that assures almost sure practical stability for the bearing angle $\alpha_{j}$. Figure 3 is used to interpret the basic idea and results in this section. Two types of sets are depicted in Figure 3 with one enclosed by the blue curve, and the other one enclosed by the red curve. The blue curve enclosed set represents the partition generated by inequality $G\left(\left|\alpha_{j}\right|,\left|L_{j}\right|\right) \leq \eta_{j}$ with associated threshold $\eta_{j} \in(0,1)$, which is shown in Lemma 4.7. The red-curve enclosed area characterizes the target set where the system trajectory will converge to almost surely. The size of the target set is characterized by $\alpha_{j}^{*}\left(\eta_{j}\right)$ which varies as a function of the threshold $\eta_{j}$.

The main result states that the bearing angle $\alpha_{j}$ will almost surely converge to the target set if the system trajectory enters and remains in the set enclosed by the blue curve. To assure the invariance of the blue curve enclosed set, we adopt a switching control strategy to reconfigure the control gain for each leader-follower pair. Figure 3 shows one possible evolution of the system trajectory $\alpha_{j}$ and $L_{j}$ with the switching strategy. We use black dots to represent the estimates of the bearing angle $\hat{\alpha}_{j}\left(\tau_{k}\right)$ at each sampling time $\tau_{k}$. A bar is used to characterize the uncertainty interval with the estimate $\hat{\alpha}_{j}\left(\tau_{k}\right)$ as its center. The length of bar can be viewed as an upper bound of the quantization error $\left|\alpha_{j}\left(\tau_{k}\right)-\hat{\alpha}_{j}\left(\tau_{k}\right)\right|$, and increases as the channel condition decreases. Therefore, the basic idea for switching is that when the system trajectory approaches the blue set's boundary with an increasing uncertainty length, an appropriate controller is re-selected to assure the stochastic variation on the uncertainty length satisfies a supermartingale like inequality, which guarantees the system states converge to the target set with probability one.

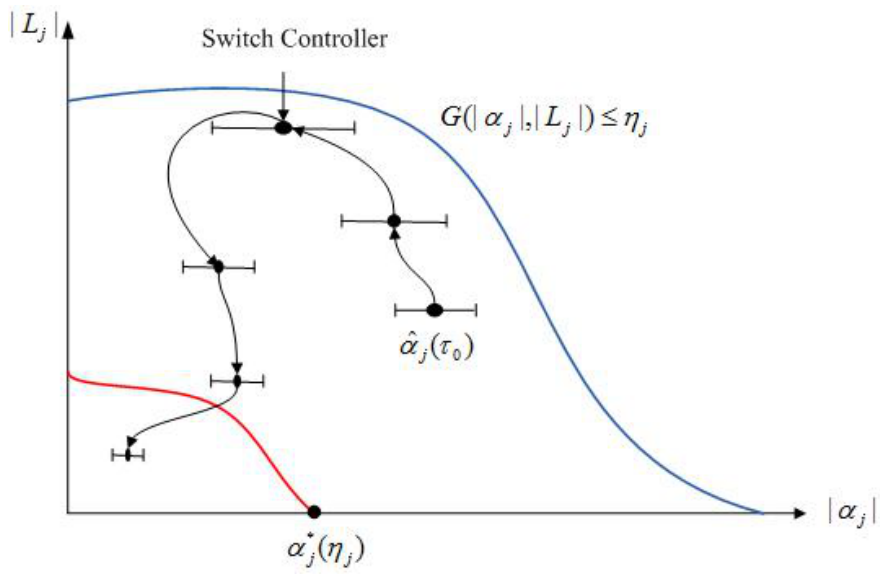

Figure 3: Partition of formation state space.

To be more specific about the main result, we first use a dynamic quantization method to show that the quantization error $\mid \alpha_{j}\left(\tau_{k}\right)-$ $\hat{\alpha}_{j}\left(\tau_{k}\right) \mid$ can be bounded by a sequence that is recursively constructed (Lemma 4.6). Then, we present a sufficient condition on the selection of controller, under which the sequence satisfies a supermartingale like inequality (Lemma 4.7). Finally, the super-martingale inequality condition leads to the proof of almost sure practical stability for the sequence (Theorem 4.9), and as well as the bearing angle $\alpha_{j}$ (Theorem 4.12).
Recall that $\left\{\alpha_{j}\left(k^{-}\right), U_{j}(k)\right\}_{k=0}^{\infty}$ characterizes the quantizer's state at each time instance $\tau_{k}$. The following lemma gives a recursive construction for this sequence such that the quantization error remains bounded by some function of $U_{j}(k)$ for all $k \geq 0$. Such predictable bound is used to switch controllers to assure almost sure performance. Note that the technique used to prove the Lemma follows the pattern in traditional dynamical quantization [15, 3].

LEMMA 4.6. Consider the closed-loop system (8-9), given the transmission time sequence $\left\{\tau_{k}\right\}_{k=0}^{\infty}$, and controller pairs $\left\{K_{L_{j}}(k)\right.$, $\left.K_{\alpha_{j}}(k)\right\}_{k=0}^{\infty}$. Let $T_{k}=\tau_{k+1}-\tau_{k}$, let the hypothesis of proposition 4.1 and Lemma 4.4 hold, the initial ordered pair $\left\{\hat{\alpha}_{j}(0), U_{j}(0)\right\}$ is known to both leader and follower, and the initial state $\alpha_{j}(0) \in$ $\left[-U_{j}(0), U_{j}(0)\right], U_{j}(0) \leq \frac{\pi}{2}$. If the sequence $\left\{\alpha_{j}\left(k^{-}\right), U_{j}(k)\right\}_{k=0}^{\infty}$ is constructed by the following recursive equation,

$$
\begin{aligned}
U_{j}(k+1) & =B_{j}(k) T_{k}+2^{-R_{j}(k)} U_{j}(k) \\
\hat{\alpha}_{j}\left(k+1^{-}\right) & =\left(\hat{\alpha}_{j}\left(k^{+}\right)-\alpha_{d_{j}}\right) e^{-K_{\alpha_{j}}(k) T_{k}}+\alpha_{d_{j}}
\end{aligned}
$$

where

$$
\begin{aligned}
B_{j}(k) & =\max \left\{\frac{1}{\min \left\{L_{j \min }, L_{j}(k)\right\}}, 1\right\} W_{j-1}\left(\left|\tilde{\alpha}_{j}(k)\right|\right) \\
L_{j \min } & =\left[-\tilde{L}_{j}(k)+\frac{W_{j-1}\left(\left|\tilde{\alpha}_{j}(k)\right|\right)}{K_{L_{j}}(k)}\right] e^{-K_{L_{j}}(k) T_{k}}+L_{d_{j}}-\frac{W_{j-1}\left(\left|\tilde{\alpha}_{j}(k)\right|\right)}{K_{L_{j}}(k)} \\
\tilde{L}_{j}(k) & =L_{d_{j}}-L_{j}(k)
\end{aligned}
$$

then the bearing angle $\alpha_{j}(k)$ for all $j=1,2, \ldots, N-1$ generated by system equations (8-9) can be bounded as

$$
\left|\alpha_{j}(k)-\hat{\alpha}_{j}\left(k^{+}\right)\right| \leq \bar{U}_{j}(k)
$$

where $\bar{U}_{j}(k)=2^{-R_{j}(k)} U_{j}(k)$ and $R_{j}(k)$ is the number of bits received over the time interval $\left[\tau_{k}, \tau_{k+1}\right)$.

Proof. Let $e_{j}(t)=\alpha_{j}(t)-\hat{\alpha}_{j}(t)$ denote the estimation error, and we consider the dynamic of $e_{j}(t)$ over time interval $\left[\tau_{k}, \tau_{k+1}\right)$,

$$
\dot{e}_{j}=\left[\begin{array}{ll}
-\frac{\sin \alpha_{j}}{L_{j}} & 1
\end{array}\right]\left[\begin{array}{c}
v_{j-1} \\
\omega_{j-1}
\end{array}\right]
$$

By inequality $\frac{d\left|e_{j}\right|}{d t} \leq\left|\frac{d e_{j}}{d t}\right|$, we have

$$
\begin{aligned}
\frac{d\left|e_{j}\right|}{d t} & \leq\left|\left[\begin{array}{ll}
-\frac{\sin \alpha_{j}}{L_{j}} & 1
\end{array}\right]\left[\begin{array}{c}
v_{j-1} \\
\omega_{j-1}
\end{array}\right]\right| \\
& \leq\left(\frac{1}{\left|L_{j}\right|}+1\right)\left|\left[\begin{array}{c}
v_{j-1} \\
\omega_{j-1}
\end{array}\right]\right| \\
& \leq\left(\frac{1}{\left|L_{j}\right|}+1\right) W_{j-1}\left(\left|\tilde{\alpha}_{j}(k)\right|\right)
\end{aligned}
$$

The last inequality holds because of Proposition 4.1. The explicit bound on $\left|L_{j}\right|$ over time interval $\left[\tau_{k}, \tau_{k+1}\right)$ can be derived as follows,

$$
\begin{aligned}
\dot{L}_{j} & \geq K_{L_{j}}(k)\left(L_{d_{j}}-L_{j}\right)-\left|v_{j-1}\right| \\
& \geq K_{L_{j}}(k)\left(L_{d_{j}}-L_{j}\right)-W_{j-1}\left(\left|\tilde{\alpha}_{j}(k)\right|\right)
\end{aligned}
$$

Using Gronwall-Bellman inequality over $\left[\tau_{k}, \tau_{k+1}\right)$ yields,

$$
\begin{aligned}
L_{j}(t) & \geq\left[L_{j}\left(\tau_{k}\right)-\left(L_{d_{j}}-\frac{W_{j-1}\left(\left|\tilde{\alpha}_{j}(k)\right|\right)}{K_{L_{j}}(k)}\right)\right] e^{-K_{L_{j}}(k)\left(t-\tau_{k}\right)} \\
& \left.+L_{d_{j}}-\frac{W_{j-1}\left(\left|\tilde{\alpha}_{j}(k)\right|\right)}{K_{L_{j}}(k)}\right)
\end{aligned}
$$


Since $L_{d_{j}} \geq \frac{W_{j-1}\left(\left|\tilde{\alpha}_{j}(k)\right|\right)}{K_{L_{j}}(k)}$ and $L_{j}(t)>d$ from Lemma 4.4, we know $\inf _{\tau_{k} \leq t<\tau_{k+1}} L_{j}(t)$ is obtained at either $t=\tau_{k}$ or $t=\tau_{k+1}$,

$$
\begin{aligned}
L_{j}(t) & \geq \inf _{\tau_{k} \leq t<\tau_{k+1}} L_{j}(t)=\min \left\{L_{j \min }, L_{j}\left(\tau_{k}\right)\right\} \\
L_{j \min } & =\left[-\tilde{L}_{j}(k)+\frac{W_{j-1}\left(\left|\tilde{\alpha}_{j}(k)\right|\right)}{K_{L_{j}}(k)}\right] e^{-K_{L_{j}}(k) T_{k}} \\
& +\left(L_{d_{j}}-\frac{W_{j-1}\left(\left|\tilde{\alpha}_{j}(k)\right|\right)}{K_{L_{j}}(k)}\right)
\end{aligned}
$$

By inequality (19), (18) is rewritten as

$$
\frac{d\left|e_{j}\right|}{d t} \leq\left(\frac{1}{\min \left\{L_{j \min }, L_{j}\left(\tau_{k}\right)\right\}}+1\right) W_{j-1}\left(\left|\tilde{\alpha}_{j}(k)\right|\right)
$$

Solving above differential inequality, we have

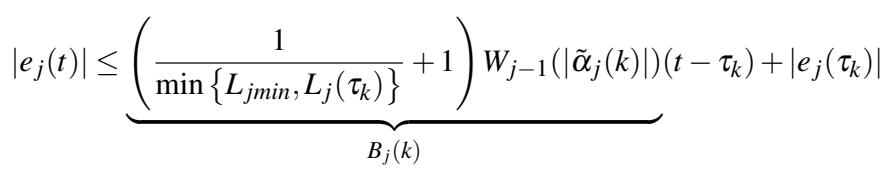

For $t \rightarrow \tau_{k+1}$, one can get $\left|e\left(k+1^{-}\right)\right| \leq B_{j}(k) T_{k}+\left|e_{j}(k)\right|$. And assume that $\left|e_{j}(k)\right| \leq \bar{U}_{j}(k)$, then $\left|e\left(k+1^{-}\right)\right| \leq B_{j}(k) T_{k}+\bar{U}_{j}(k)$. We know that

$\left|e\left(k+1^{+}\right)\right| \leq 2^{-R_{j}(k+1)}\left|e\left(k+1^{-}\right)\right| \leq 2^{-R_{j}(k+1)}\left(B_{j}(k) T_{k}+\bar{U}_{j}(k)\right)$

From equation (14) and $\bar{U}_{j}(k+1)=2^{-R_{j}(k+1)} U_{j}(k+1)$, we have $\left|e\left(k+1^{+}\right)\right| \leq \bar{U}_{j}(k+1)$. The equation (15) holds by simply considering the solution to the ODE $\dot{\tilde{\alpha}}_{j}=-K_{\alpha_{j}} \tilde{\alpha}_{j}$ with initial value $\tilde{\alpha}_{j}=\alpha_{d_{j}}-\hat{\alpha}_{j}\left(k^{+}\right)$.

With Lemma 4.6, the following lemma provides a sufficient condition on the selection of controller gains that leads to almost-surely practical stability for the bearing angle $\alpha_{j}, j=1,2, \ldots, N-1$.

LEMMA 4.7. Consider the closed loop system in equations (89). Let

$G\left(\left|\alpha_{j}\right|,\left|L_{j}\right|\right)=e^{-h\left(\left|\alpha_{j}\right|,\left|L_{j}\right|\right) \gamma\left(\left|\alpha_{j}\right|,\left|L_{j}\right|\right)}\left(1+h\left(\left|\alpha_{j}\right|,\left|L_{j}\right|\right) \gamma\left(\left|\alpha_{j}\right|,\left|L_{j}\right|\right)\right)$

be non-negative, monotone increasing function with respect to $\left|\alpha_{j}\right|$ and $\left|L_{j}\right|$ respectively. If there exists a sequence of controller gains $\left\{K_{L_{j}}(k), K_{\alpha_{j}}(k)\right\}_{k=0}^{\infty}$ with $K_{j}(k)=\left\{K_{L_{j}}(k), K_{\alpha_{j}}(k)\right\} \in \mathscr{K}_{j}$ for all $k \in \mathbb{Z}$ such that the Proposition 4.1 and following inequality hold for any $\eta_{j} \in(0,1)$

$$
G\left(\bar{\alpha}_{j}(k+1), \bar{L}_{j}(k+1)\right) \leq \eta_{j}
$$

$\bar{\alpha}_{j}(k+1)=\left|-\tilde{\alpha}_{j}(k) e^{-K_{\alpha_{j}}(k) T_{k}}+\alpha_{d_{j}}\right|+B_{j}(k) T_{k}+\bar{U}_{j}(k)$

$\bar{L}_{j}(k+1)=L_{d_{j}}+\frac{W_{j-1}\left(\left|\tilde{\alpha}_{j}(k)\right|\right)}{K_{L_{j}}(k)}-\left[\tilde{L}_{j}(k)+\frac{W_{j-1}\left(\left|\tilde{\alpha}_{j}(k)\right|\right)}{K_{L_{j}}(k)}\right] e^{-K_{L_{j}}(k) T_{k}}$

then

$$
\mathbb{E}\left[\bar{U}_{j}(k+1) \mid \bar{U}_{j}(k)\right] \leq \eta_{j} \bar{U}_{j}(k)+\eta_{j} B_{j}(k) T_{k}, \forall k \in \mathbb{Z}_{+}
$$

Proof. Consider the sequence $\left\{\bar{U}_{j}(k)\right\}_{k=0}^{\infty}$ that satisfies equation (14) in Lemma 4.6, using the argument in [7], the conditional expectation $\mathbb{E}\left[\bar{U}_{j}(k+1) \mid \bar{U}_{j}(k)\right]$ can be bounded as

$\mathbb{E}\left[\bar{U}_{j}(k+1) \mid \bar{U}_{j}(k)\right] \leq G\left(\left|\alpha_{j}(k+1)\right|,\left|L_{j}(k+1)\right|\right)\left(B_{j}(k) T_{k}+\bar{U}_{j}(k)\right)$

Let $G\left(\left|\alpha_{j}(k+1)\right|,\left|L_{j}(k+1)\right|\right) \leq \eta_{j}$, we have final conclusion (21) hold. In order to select the controller gain $\left\{K_{L_{j}}(k), K_{\alpha_{j}}(k)\right\}$ for the time interval $\left[\tau_{k}, \tau_{k+1}\right)$, the selection decision is made based only on the information at time instant $\tau_{k}$. Thus, we further bound the state $\left|\alpha_{j}(k+1)\right|$ and $\left|L_{j}(k+1)\right|$ by considering

$$
\begin{aligned}
\left|e_{j}\left(k+1^{-}\right)\right| & =\left|\alpha_{j}\left(k+1^{-}\right)-\hat{\alpha}_{j}\left(k+1^{-}\right)\right| \\
& \leq U_{j}(k+1)=B_{j}(k) T_{k}+\bar{U}_{j}(k)
\end{aligned}
$$

since $\alpha_{j}(k+1)=\alpha_{j}\left(k+1^{-}\right)$, we have

$$
\begin{aligned}
\left|\alpha_{j}(k+1)\right| & \leq\left|\hat{\alpha}_{j}\left(k+1^{-}\right)\right|+B_{j}(k) T_{k}+\bar{U}_{j}(k) \\
& \leq\left|\alpha_{d_{j}}-\left(\alpha_{d_{j}}-\hat{\alpha}_{j}(k)\right) e^{-K_{L_{j}}(k) T_{k}}\right|+B_{j}(k) T_{j}+\bar{U}_{j}(k) \\
& \triangleq \bar{\alpha}_{j}(k+1)
\end{aligned}
$$

Similarly, we can also bound $\left|L_{j}(k+1)\right|$ by $\bar{L}_{j}(k+1)$ that is shown in Proposition 4.1,

$$
\begin{aligned}
\left|L_{j}(k+1)\right| \leq \bar{L}_{j}(k+1) & =\left(L_{d_{j}}+\frac{W_{j-1}\left(\left|\tilde{\alpha}_{j}(k)\right|\right)}{K_{L_{j}}(k)}\right)\left(1-e^{-K_{L_{j}}(k) T_{k}}\right) \\
& +L_{j}(k) e^{-K_{L_{j}}(k) T_{k}}
\end{aligned}
$$

Since the function $G\left(\left|\alpha_{j}(k+1)\right|,\left|L_{j}(k+1)\right|\right)$ is monotone increasing function w.r.t $\left|\alpha_{j}(k+1)\right|$ and $\left|L_{j}(k+1)\right|$, then if

$$
G\left(\bar{\alpha}_{j}(k+1), \bar{L}_{j}(k+1)\right) \leq \eta_{j}
$$

we have

$$
G\left(\left|\alpha_{j}(k+1)\right|,\left|L_{j}(k+1)\right|\right) \leq \eta_{j}
$$

then the final conclusion holds.

REMARK 4.8. Function $G\left(\alpha_{j}, L_{j}\right)$ in condition (20) is directly related to the EBB model, and it generates a partition of the formation state space as shown in Figure 3. Each partition associates with a threshold $\eta_{j}$ that characterizes the convergent rate for the uncertainty set. The aim of switching control strategy is to guarantee the condition (20) holds with a selected $\eta_{j}$.

With Lemma 4.6, the following theorem proves that sequence $\left\{\bar{U}_{j}(k)\right\}_{k=0}^{\infty}$ is almost-surely convergent to a compact set.

THEOREM 4.9. Suppose the sequence $\left\{\bar{U}_{j}(k)\right\}_{k=0}^{\infty}$ is generated by equation (14) in Lemma 4.6, if there exists a sequence of controller pairs $\left\{K_{L_{j}}(k), K_{\alpha_{j}}(k)\right\}_{k=0}^{\infty}$ such that inequality (21) holds with $\eta_{j}(k)=\eta_{j} \in(0,1)$ for all $k=0,1, \ldots, \infty$, then we have

$\lim _{k \rightarrow \infty} \operatorname{Pr}\left\{\bar{U}_{j}(k)-U_{j m}^{*}>\varepsilon\right\} \leq \lim _{k \rightarrow \infty}\left(\frac{\eta_{j}^{k} U_{j}(0)}{\varepsilon}-\frac{\eta_{j}^{k+1}}{\left(1-\eta_{j}\right) \varepsilon} B_{j}^{*} T^{*}\right)$ $=0$

with $U_{j m}^{*}=\frac{\eta_{j}}{1-\eta_{j}} B_{j}^{*} T^{*}$, where $B_{j}^{*}=\sup _{k}\left\{B_{j}(k)\right\}$ and $T^{*}=\sup _{k}\left\{T_{k}\right\}$.

PROOF. Consider the inequality (21), let $B_{j}^{*}=\sup _{k}\left\{B_{j}(k)\right\}$ and $T^{*}=\sup _{k}\left\{T_{k}\right\}$, then

$$
\mathbb{E}\left[\bar{U}_{j}(k+1) \mid \bar{U}_{j}(k)\right] \leq \eta_{j}(k) \bar{U}_{j}(k)+\eta_{j}(k) B_{j}^{*} T^{*}
$$

Taking the expectation on both side of above inequality and propagating backward, yields

$$
\begin{aligned}
\mathbb{E}\left[\bar{U}_{j}(k+1)\right] & \leq \eta_{j}^{k+1} \bar{U}_{j}(0)+\sum_{i=1}^{k+1} \eta_{j}^{i} B_{j}^{*} T^{*} \\
& =\eta_{j}^{k+1} \bar{U}_{j}(0)+\frac{\eta_{j}\left(1-\eta_{j}^{k+1}\right)}{1-\eta_{j}} B_{j}^{*} T^{*}
\end{aligned}
$$


then, using Markov inequality, we have for all $\varepsilon>0$

$$
\begin{aligned}
\lim _{k \rightarrow \infty} \operatorname{Pr}\left\{\bar{U}_{j}(k)-U_{j m}^{*}>\varepsilon\right\} & \leq \lim _{k \rightarrow \infty} \frac{\mathbb{E}\left[\bar{U}_{j}(k)-U_{j m}^{*}\right]}{\varepsilon} \\
& \leq \lim _{k \rightarrow \infty}\left(\frac{\eta_{j}^{k} U_{j}(0)}{\varepsilon}-\frac{\eta_{j}^{k+1}}{\left(1-\eta_{j}\right) \varepsilon} B_{j}^{*} T^{*}\right) \\
& =0
\end{aligned}
$$

REMARK 4.10. From Lemma 4.6, one knows that $B_{j}^{*}=\frac{\sup _{k}\left\{W_{j-1}\left(\tilde{\alpha}_{j}(k)\right)\right\}}{d}$. With $U_{j m}^{*}=\frac{\eta_{j}}{1-\eta_{j}} B_{j}^{*} T^{*}$, one may have smaller $U_{j m}^{*}$ by reducing the sampling period $T *$, function gain $W_{j-1}$ or threshold $\eta_{j}$.

REMARK 4.11. Though the bound $U_{j m}^{*}$ on the limit set is conservative since the proof is based on the worst case of $B_{j}(k), T_{k}$ and $W_{j-1}$, the fundamental relationship between $U_{j m}$ and $B_{j}(k), T_{k}, W_{j-1}$ still holds for tight bounds.

With Theorem 4.9, we proceed to state the main theorem as follows,

THEOREM 4.12. Consider the closed-loop system in equations (8-9), if there exists a sequence of controller pair $\left\{K_{L_{j}}(k), K_{\alpha_{j}}(k)\right\}_{k=0}^{\infty}$ such that the hypothesis of Theorem 4.9 holds, then there exists a positive bounded real number $\alpha_{j}^{*}$, the random process $\left\{\alpha_{j}(t)\right\}, t \in$ $\mathbb{R}_{+}$is almost-surely convergent to a compact set $\left\{\alpha_{j} \in \mathbb{R} \| \alpha_{d_{j}}-\right.$ $\left.\alpha_{j} \mid \leq \alpha_{j}^{*}\right\}$, i.e.

$$
\lim _{t \rightarrow \infty} \operatorname{Pr}\left\{\sup _{k>t}\left|\alpha_{d_{j}}-\alpha_{j}(k)\right|-\alpha_{j}^{*}>\varepsilon\right\}=0, \forall \varepsilon>0
$$

where $\alpha_{j}^{*}=\frac{B_{j}^{*} T^{*}}{1-\eta_{j}}\left(\eta_{j}+\frac{1}{1-e^{-K_{\alpha_{j}}^{*} T_{\min }}}\right)$.

Proof. Consider $t \in\left[\tau_{k}, \tau_{k+1}\right)$, we know

$$
\dot{\hat{\alpha}}_{j}=K_{\alpha_{j}}(k)\left(\alpha_{d_{j}}-\hat{\alpha}_{j}(t)\right)
$$

with initial value $\hat{\alpha}_{j}\left(\tau_{k}\right)$. Therefore, let $\tilde{\alpha}_{j}(k)=\alpha_{d_{j}}-\hat{\alpha}_{j}(k)$, we have

$$
\tilde{\alpha}_{j}\left(k+1^{-}\right)=e^{-K_{\alpha_{j}}(k) T_{k}} \tilde{\alpha}_{j}(k)
$$

Let $E_{j}(k+1)=\tilde{\alpha}_{j}(k+1)-\tilde{\alpha}_{j}\left(k+1^{-}\right)$, then

$$
\tilde{\alpha}_{j}(k+1)=e^{-K_{\alpha_{j}}(k) T_{k}} \tilde{\alpha}_{j}(k)+E_{j}(k+1)
$$

Let $K_{\alpha_{j}}^{*}=\min \left\{K_{\alpha_{j}} \mid K_{\alpha_{j}} \in \mathscr{K}_{j}\right\}$ and $T_{\min }=\min \left\{T_{k} \mid k \in \mathbb{Z}_{+}\right\}$

$$
\left|\tilde{\alpha}_{j}(k+1)\right| \leq e^{-K_{\alpha_{j}}^{*} T_{\min }}\left|\tilde{\alpha}_{j}(k)\right|+\left|E_{j}(k+1)\right|
$$

The term $\left|E_{j}(k+1)\right|$ can be further bounded by

$$
\begin{aligned}
\left|E_{j}(k+1)\right| & \leq\left(B_{j}(k) T_{k}+\bar{U}_{j}(k)\right)\left(1-2^{-R_{j}(k+1)}\right) \\
& \leq B_{j}^{*} T^{*}+\bar{U}_{j}(k)
\end{aligned}
$$

Taking the expectation on both sides of inequality (23) and using above bound on $|E(k+1)|$ yields

$$
\mathbb{E}\left[\left|\tilde{\alpha}_{j}(k+1)\right|\right] \leq e^{-K_{\alpha_{j}}^{*} T_{\min }} \mathbb{E}\left[\left|\tilde{\alpha}_{j}(k)\right|\right]+B_{j}^{*} T^{*}+\mathbb{E}\left[\bar{U}_{j}(k)\right]
$$

Propagating backward above inequality and with the result from Theorem 4.9, we have

$$
\begin{aligned}
& \mathbb{E}\left[\left|\tilde{\alpha}_{j}(k+1)\right|\right] \\
& \leq e^{-K_{\alpha_{j}}^{*} T_{\min }(k+1)} \mathbb{E}\left[\left|\tilde{\alpha}_{j}(0)\right|\right]+\frac{1-e^{-(k+1) K_{\alpha_{j}}^{*} T_{\min }}}{1-e^{-K_{\alpha_{j}} T_{\min }}} B_{j}^{*} T^{*} \\
& +\sum_{i=0}^{k+1} e^{-i K_{\alpha_{j}}^{*} T_{\min }} \mathbb{E}\left[\bar{U}_{j}(k-i)\right] \\
& \leq e^{-K_{\alpha_{j}}^{*} T_{\text {min }}(k+1)} \mathbb{E}\left[\left|\tilde{\alpha}_{j}(0)\right|\right]+\frac{1-e^{-(k+1) K_{\alpha_{j}}^{*} T_{\text {min }}}}{1-e^{-K_{\alpha_{j}} T_{\min }}} B_{j}^{*} T^{*} \\
& +\frac{\eta_{j}}{1-\eta_{j}} B_{j}^{*} T^{*}\left[\frac{1-e^{-(k+1) K_{\alpha_{j}}^{*} T_{\min }}}{1-e^{-K_{\alpha_{j}}^{*} T_{\min }}}-\frac{\eta_{j}^{k+1}-e^{-(k+1) K_{\alpha_{j}}^{*} T_{\min }}}{\eta_{j}-e^{-K_{\alpha_{j}}^{*} T_{\min }}}\right] \\
& +\frac{\eta_{j}^{k+1}-e^{-(k+1) K_{\alpha_{j}}^{*} T_{\min }}}{\eta_{j}-e^{-K_{\alpha_{j}}^{*} T_{\min }}} \bar{U}_{j}(0) \\
& \triangleq g(k)+\frac{B_{j}^{*} T^{*}}{\left(1-\eta_{j}\right)\left(1-e^{-K_{\alpha_{j}}^{*} T_{\min }}\right)}
\end{aligned}
$$

Because

$$
\begin{aligned}
& \mathbb{E}\left[\bar{U}_{j}(k+1)\right] \\
& \leq \eta_{j}^{k+1} \bar{U}_{j}(0)+\frac{\eta_{j}\left(1-\eta_{j}^{k+1}\right)}{1-\eta_{j}} B_{j}^{*} T^{*} \\
& =g_{2}(k)+\frac{\eta_{j}}{1-\eta_{j}} B_{j}^{*} T^{*}
\end{aligned}
$$

where $g_{i}(k), i=1,2$ are exponentially decaying functions with $\lim _{k \rightarrow \infty} g_{i}(k)=0, i=1,2$, and $\mathbb{E}\left[\left|\alpha_{d_{j}}-\alpha_{j}(k)\right|\right]=\mathbb{E}\left[\left|\tilde{\alpha}_{j}(k)+\bar{U}_{j}(k)\right|\right] \leq$ $\mathbb{E}\left[\mid \tilde{\alpha}_{j}(k)\right]+\mathbb{E}\left[\bar{U}_{j}(k) \mid\right]$, then

$$
\begin{aligned}
& \lim _{t \rightarrow \infty} \operatorname{Pr}\left\{\sup _{k>t}\left|\alpha_{d_{j}}-\alpha_{j}(k)(k)\right|-\alpha_{j}^{*}>\varepsilon\right\} \\
& \leq \lim _{t \rightarrow \infty} \frac{\mathbb{E}\left[\mid \tilde{\alpha}_{j}(k)\right]+\mathbb{E}\left[\bar{U}_{j}(k) \mid\right]-\alpha_{j}^{*}}{\varepsilon} \\
& \leq \lim _{t \rightarrow \infty} \frac{g_{1}(k)+g_{2}(k)}{\varepsilon}=0
\end{aligned}
$$

REMARK 4.13. The inequality (24) holds if the hypothesis of Markov inequality holds for our cases. Though we are still working on a rigorous proof to confirm that hypothesis, we believe the final results hold.

\section{SIMULATION EXPERIMENTS}

This section presents simulation experiments examining the resilience of our proposed switched controller to deep fades, and also demonstrates the benefits of using almost sure practical stability as a safety measurement over the traditional mean square stability.

\subsection{Simulation Setup}

In the simulation, we consider $N=4$ vehicles that is cascaded in a string as shown in Figure 1. Each leader-follower pair uses a two-state Markov chain model to simulate the fading channel between the leader and follower. The two-state Markov chain has two states with one representing the good channel condition and the other one representing the bad channel condition. Here, the 
"good channel state" simply means the transmitted bit is successfully received, while the "bad channel state" means the failure of receiving the bit.

Following the characterization of Makov chain model in [18], one can find that the conditional probability for good channel state is a monotone decreasing function of $\frac{L_{j}(t)}{\cos \alpha_{j}(t)}$, while the conditional probability for bad channel state is a monotone decreasing function of $\frac{\cos \alpha_{j}(t)}{L_{j}(t)}$. The explicit function form depends on the distribution of the channel gain. In this simulation, we therefore use $p_{11}=e^{-3 \times 10^{-3}\left(\frac{L_{j}(t)}{\cos \alpha_{j}(t)}\right)^{2}}$ to denote the conditional probability for the good channel state. Let $p_{22}=e^{-6 \times 10^{2}\left(\frac{\cos \alpha_{j}(t)}{L_{j}(t)}\right)^{2}}$ represent the conditional probability for the bad channel condition. Hence, the corresponding transition probabilities between these states are $1-p_{11}$ and $1-p_{22}$. Then, we utilize the EBB model in equation (6) to characterize the low bit region generated by the two-state markov chain model. The corresponding functions in EBB model (6) are

$$
h\left(\alpha_{j}, L_{j}\right)=\bar{R}_{j} e^{-3 \times 10^{-4}\left(\frac{L_{j}(t)}{\cos \alpha_{j}(t)}\right)^{2}}, \gamma\left(\alpha_{j}, L_{j}\right)=e^{-4.5 \times 10^{-3}\left(\frac{L_{j}(t)}{\cos \alpha_{j}(t)}\right)^{2}}
$$

with $\bar{R}_{j}=2$ representing two bits are transmitted at each sampling period.

The $100 \mathrm{~ms}$ sampling time that is widely used in mobile robot system, is selected for each leader-follower pair $(j=1,2,3)$, i,e, $T_{k}=0.1 \mathrm{sec}$ for all $k \in \mathbb{Z}_{+}$. The functions $W_{j-1}(\cdot)$ in Proposition 4.1 are selected to be linear functions $W_{j-1}\left(\left|\tilde{\alpha}_{j}(t)\right|\right)=a_{j}\left|\tilde{\alpha}_{j}(t)\right|+$ $b_{j}$ with parameters selected as follows

$$
a_{1}=0.1, b_{1}=0.01 ; a_{2}=0.8, b_{2}=2 ; a_{3}=1, b_{3}=4
$$

The value of the parameter sets are chosen to be increasing with respect to $j$ to guarantee the feasibility of the controller selection for each leader-follower system.

In this simulation, we consider an interesting and realistic scenario that the fourth vehicle from far distance intends to merge into the other three closed-clustered vehicles. Hence, the initial states for three leader-follower pairs $(j=1,2,3)$ are set as

$$
\alpha_{1}\left(\tau_{0}\right)=\frac{\pi}{3}, \alpha_{2}\left(\tau_{0}\right)=\frac{\pi}{4}, \alpha_{3}\left(\tau_{0}\right)=\frac{\pi}{6} .
$$

with initial uncertainty length $U_{j}\left(\tau_{0}\right)=\frac{\pi}{6}$, and

$$
L_{1}\left(\tau_{0}\right)=7.1, L_{2}\left(\tau_{0}\right)=7.1, L_{3}\left(\tau_{0}\right)=99 .
$$

By switching controller pairs from the following pool

$$
\mathscr{K}_{j}=\left\{\left(K_{L_{j}}, K_{\alpha_{j}}\right): 0<K_{L_{j}} \leq 100,0<K_{\alpha_{j}} \leq 100\right\} .
$$

each leader-follower pair is required to achieve and maintain around desired setpoints $\alpha_{d_{j}}=0, L_{d_{j}}=2, j=1,2,3$.

\subsection{Simulation Results}

A Monte Carlo method was used to verify that the system has almost surely practical stability when Proposition 4.1 and Lemma 4.7 hold. Each simulation example is run 100 times over a time interval from 0 to 10 seconds.

In the first simulation, we select the controllers for each leaderfollower pair from $\mathscr{K}_{j}, j=1,2,3$ so that Proposition 4.1 and Lemma 4.7 hold at each time instant $\tau_{k}$. Figures $4-5$ show the maximum and minimum values of the system states $L_{j}$ and $\alpha_{j}, j=1,2,3$ evaluated over all the 100 runs. In both figures, the maximum value is marked by red lines and the minimum value is marked by blue lines. The two dashed lines in Figure 5 represent the upper and lower bound for the relative bearing $\alpha$, i.e. $\left|\alpha_{j}\right| \leq \pi / 2$, which characterizes the safety region. We can see from Figures 4-5 that the maximum and minimum values of the system states asymptotically converge to a bounded set containing the desired set-points $\alpha_{d_{j}}=0$ and $L_{d_{j}}=2$. This is precisely the behavior that one would expect if the system is almost sure practical stable. These results therefore, seem to confirm our statement in Theorem 4.12. Figures 6-7
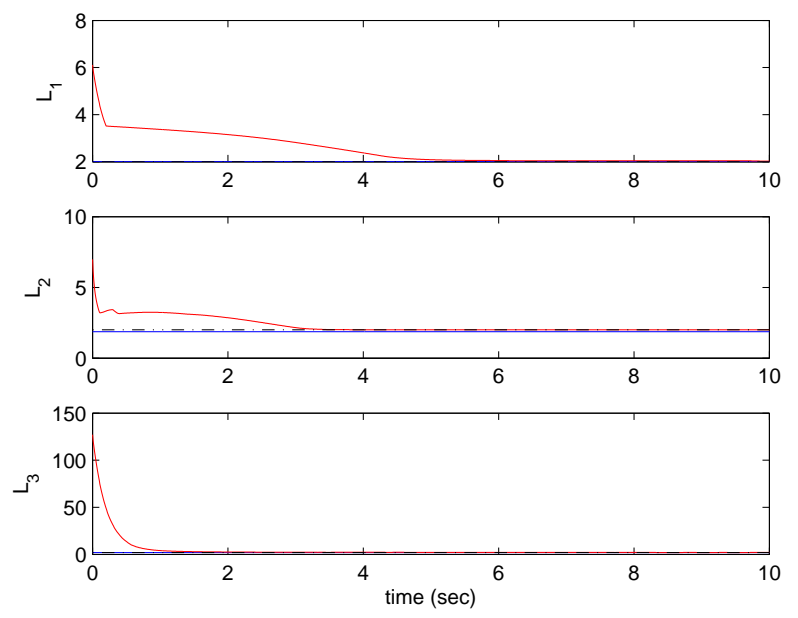

Figure 4: The maximum and minimum value of inter-vehicle distance $L_{j}$ for leader-follower pair, $j=1,2,3$.
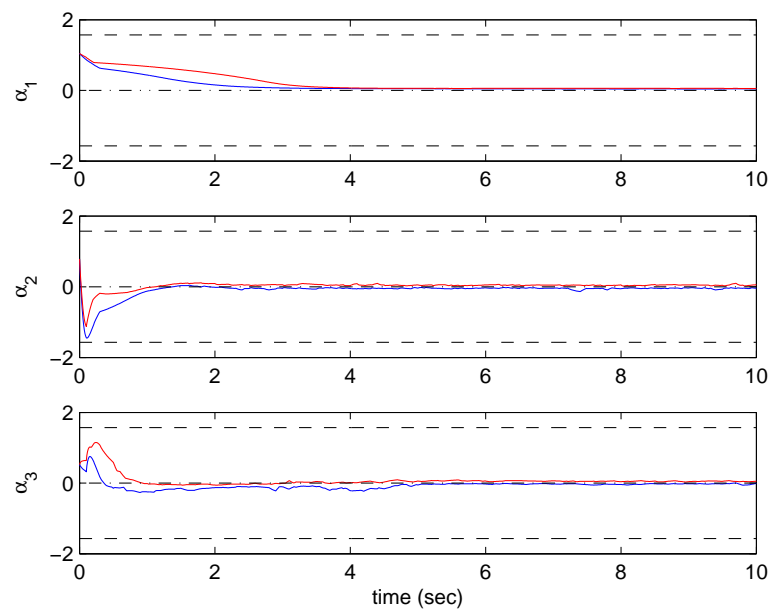

Figure 5: The maximum and minimum value of bearing angle $\alpha_{j}$ for leader-follower pair, $j=1,2,3$.

show one sample of switching controller profile and channel state for each leader-follower pair. The top plot in Figure 6 shows the switching controller profile for the leader-follower pair 1 with red line marked as controller gain $K_{\alpha_{1}}$ and blue line as controller gain $K_{L_{1}}$. The bottom one is the switching controller profile for leaderfollower pair 2 with the same marking rule. These plots show that the controller gains stay low at the first two seconds to avoid causing large disturbance to the bottom system, then switch from low to high when the systems approach the equilibrium and are confident that the channel state will always stay good. The top plot in Figure 7 is the switching controller profile for the leader-follower system 3 with same marking rule, and the bottom plot is the channel state 
$R_{3}(k)$ that characterizes the number of successfully received bits at each time interval. We can clearly see from the plots that the controller for system 3 starts with low gains to compensate the effect caused by a short string of zero bits at the beginning, and then switches from low gain to high gain when channel condition stays good. These results demonstrate that channel state indeed is used as a feedback signal to switch the controller. In the second simu-
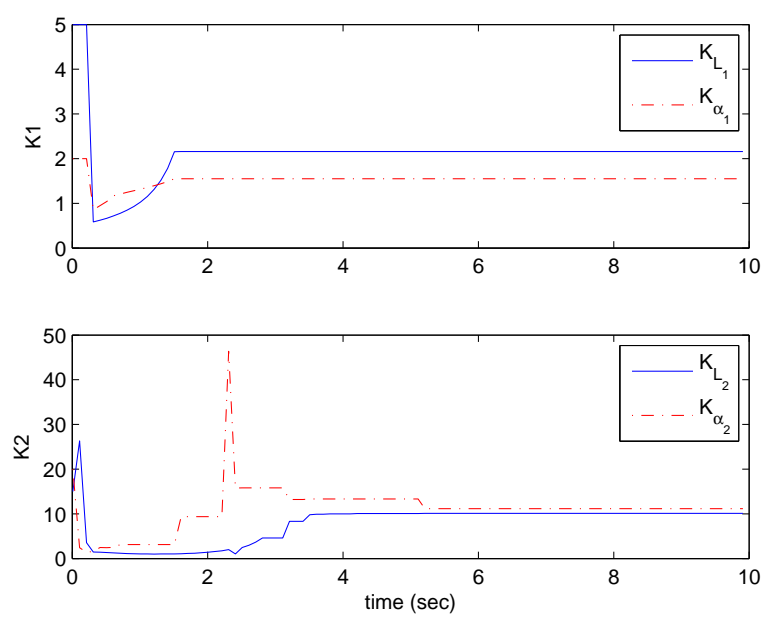

Figure 6: One sample of switching controller profile for leaderfollower pair 1 and 2
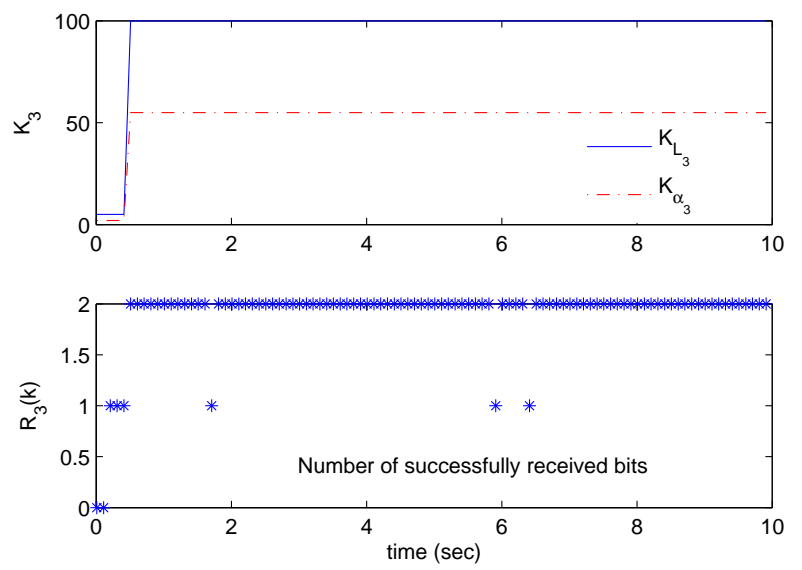

Figure 7: One sample of switching profile and channel state for leader-follower pair 3

lation, we studied the benefits of almost sure practical stability as a safety measurement over the traditional mean square stability. Traditional mean square stability requires the second moment of the system state converges to a positive constant value, but it does not put any constraint on the sample path which might potentially cause safety issues. For a fair comparison, the same simulation setup and parameters are applied in this simulation with the only difference on the controllers. One type of controller used in this simulation is a mean square stabilizing controller, which is selected to guarantee mean square stability for each leader-follower pair. The other type of controller is the switching controller proposed in this paper to guarantee almost sure practical stability for each leader-follower pair. The switching control strategy uses the mean square stabilizing controller as its initial controller.
Figure 8 shows a comparison of the maximum and minimum values of the bearing angle $\alpha_{3}$ for leader-follower pair 3 with the switching controller case in the top plot and the mean square controller $K_{1}=(5,0.5) ; K_{2}=(5,0.5) ; K_{3}=(2,50)$ in the bottom plot. It is worth noting that $\left(K_{1}, K_{2}, K_{3}\right)$ is just one of the many selections in our simulation. Because of the space limitation, we only use $\left(K_{1}, K_{2}, K_{3}\right)$ as an example to demonstrate the results. It is clear from Figure 8 that the system's sample path goes unbounded as time increases by using a mean square stabilizing controller, but it converges asymptotically to a bounded set by using a switching controller. These results suggest that the composition of mean square stable systems does not guarantee mean square stability for the whole system, while the composition of almost sure stable systems may still guarantee almost sure stability for the whole system.
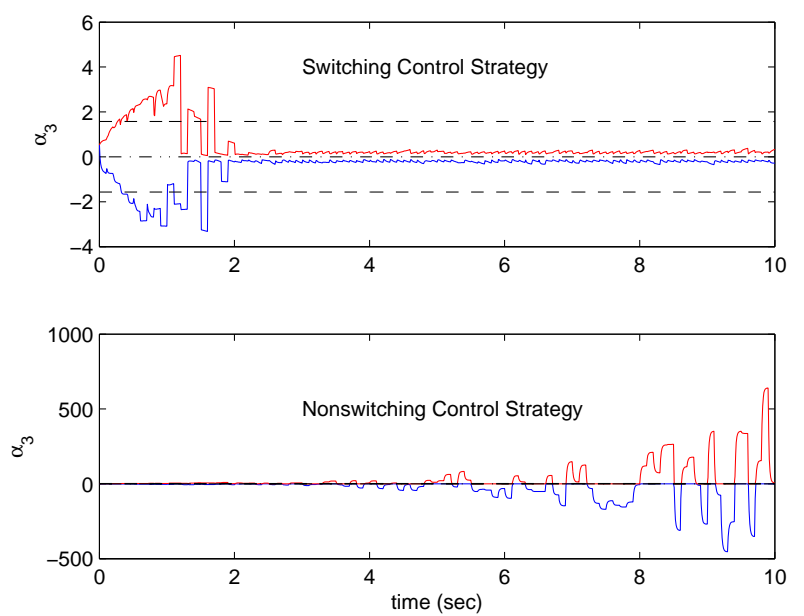

Figure 8: The maximum and minimum system trajectory for leader-follower pair 3 with switching controller (Top) and nonswitching controller pair(Bottom) $K_{L_{3}}=2$ and $K_{\alpha_{3}}=50$

\section{CONCLUSIONS}

This paper studied the almost sure practical stability for leaderfollower formation control of a class of nonholonomic system in the presence of deep fades exhibiting exponentially bounded burstiness. The main results are the sufficient conditions to select distributed switching controller to assure almost sure practical stability. The simulation results support our theoretical analysis and also illustrate the benefit of using almost surely practical stability as a safety measurement over traditional mean square stability.

\section{Acknowledgments}

The authors acknowledge the partial financial support of the National Science Foundation (NSF-CNS-1239222).

\section{REFERENCES}

[1] C. A. Balanis. Antenna theory: analysis and design/Constantine A. Balanis. J. Wiley, New York, 1982.

[2] T. Balch and R. C. Arkin. Behavior-based formation control for multirobot teams. Robotics and Automation, IEEE Transactions on, 14(6):926-939, 1998.

[3] R. W. Brockett and D. Liberzon. Quantized feedback stabilization of linear systems. Automatic Control, IEEE Transactions on, 45(7):1279-1289, 2000. 
[4] L. Cheng, B. E. Henty, D. D. Stancil, F. Bai, and P. Mudalige. Mobile vehicle-to-vehicle narrow-band channel measurement and characterization of the $5.9 \mathrm{ghz}$ dedicated short range communication (dsrc) frequency band. IEEE Journal on Selected Areas in Communications, 25(8):1501-1516, 2007.

[5] J. P. Desai, J. Ostrowski, and V. Kumar. Controlling formations of multiple mobile robots. In Robotics and Automation, 1998. Proceedings. 1998 IEEE International Conference on, volume 4, pages 2864-2869. IEEE, 1998.

[6] N. Elia and S. K. Mitter. Stabilization of linear systems with limited information. IEEE Transactions on Automatic Control, 46(9):1384-1400, 2001.

[7] B. Hu and M. D. Lemmon. Using channel state feedback to achieve resilience to deep fades in wireless networked control systems. In Proceedings of the 2nd international conference on High Confidence Networked Systems, April 9-11 2013.

[8] H. Kushner. Stochastic Stability and Control. Academic Press, 1967.

[9] M. Mesbahi and M. Egerstedt. Graph theoretic methods in multiagent networks. Princeton University Press, 2010.

[10] P. Minero, M. Franceschetti, S. Dey, and G. N. Nair. Data rate theorem for stabilization over time-varying feedback channels. IEEE Transactions on Automatic Control, 54(2):243-255, 2009.

[11] N. Moshtagh and A. Jadbabaie. Distributed geodesic control laws for flocking of nonholonomic agents. Automatic Control, IEEE Transactions on, 52(4):681-686, 2007.

[12] R. Olfati-Saber, J. A. Fax, and R. M. Murray. Consensus and cooperation in networked multi-agent systems. Proceedings of the IEEE, 95(1):215-233, 2007.

[13] D. J. Stilwell and B. E. Bishop. Platoons of underwater vehicles. Control Systems, IEEE, 20(6):45-52, 2000.

[14] D. M. Stipanović, G. I?nalhan, R. Teo, and C. J. Tomlin. Decentralized overlapping control of a formation of unmanned aerial vehicles. Automatica, 40(8):1285-1296, 2004.

[15] S. Tatikonda and S. Mitter. Control under communication constraints. IEEE Transactions on Automatic Control, 49(7):1056-1068, 2004.

[16] D. Tse and P. Viswanath. Fundamentals of wireless communication. Cambridge university press, 2005.

[17] P. Varaiya. Smart cars on smart roads: problems of control. Automatic Control, IEEE Transactions on, 38(2):195-207, 1993.

[18] H. Wang and N. Moayeri. Finite-state markov channel - a useful model for radio communication channels. IEEE Transactions on Vehicular Technology, 44(1):163-171, 1995.

[19] W. S. Wong and R. W. Brockett. Systems with finite communication bandwidth constraints. ii. stabilization with limited information feedback. IEEE Transactions on Automatic Control, 44(5):1049-1053, 1999.

[20] S. Yi, Y. Pei, and S. Kalyanaraman. On the capacity improvement of ad hoc wireless networks using directional antennas. In International Symposium on Mobile Ad Hoc Networking \& Computing: Proceedings of the 4 th ACM international symposium on Mobile ad hoc networking \& computing, volume 1, pages 108-116. Citeseer, 2003.

\section{APPENDIX}

Proof of Proposition 4.1:

Proof. Consider the infinite norm of the control input given in equation (8),

$$
\begin{aligned}
\left|\left[\begin{array}{c}
v_{j}(t) \\
\omega_{j}(t)
\end{array}\right]\right| & =\left|\left[\begin{array}{cc}
-\cos \phi_{j} & -L_{j} \sin \phi_{j} \\
-\frac{\sin \phi_{j}}{d} & \frac{L_{j}}{d} \cos \phi_{j}
\end{array}\right]\left[\begin{array}{c}
K_{L_{j}}(k)\left(L_{d_{j}}-L_{j}\right) \\
K_{\alpha_{j}}(k)\left(\alpha_{d_{j}}-\hat{\alpha_{j}}\right)
\end{array}\right]\right| \\
& \leq\left\|\left[\begin{array}{cc}
-\cos \phi_{j} & -L_{j} \sin \phi_{j} \\
-\frac{\sin \phi_{j}}{d} & \frac{L_{j}}{d} \cos \phi_{j}
\end{array}\right]\right\|\left|\left[\begin{array}{c}
K_{L_{j}}(k)\left(L_{d_{j}}-L_{j}\right) \\
K_{\alpha_{j}}(k)\left(\alpha_{d_{j}}-\hat{\alpha}_{j}\right)
\end{array}\right]\right| \\
& \leq\left(1+\left|L_{j}(t)\right|\right) \max \left\{K_{L_{j}}(k)\left|\tilde{L}_{j}(t)\right|, K_{\alpha_{j}}(k)\left|\tilde{\alpha}_{j}(t)\right|\right\}
\end{aligned}
$$

with $\tilde{L}_{j}(t)=L_{d_{j}}-L_{j}(t)$. The supreme of $\left|L_{j}(t)\right|$ over time interval $\left[\tau_{k}, \tau_{k+1}\right)$ can be obtained by considering

$$
\dot{L}_{j}(t) \leq K_{L_{j}}(k)\left(L_{d_{j}}-L_{j}\right)(t)+W_{j-1}\left(\left|\tilde{\alpha}_{j}(k)\right|\right)
$$

Using Gronwall Bellman theorem to solve above inequality and yield,

$$
\begin{aligned}
L_{j}(t) & \leq L_{j}(k) e^{-K_{L_{j}}(k)\left(t-\tau_{k}\right)}+\left(L_{d_{j}}+\frac{W_{j-1}\left(\left|\alpha_{j}(k)\right|\right)}{K_{L_{j}}(k)}\right)\left(1-e^{-K_{L_{j}}(k)\left(t-\tau_{k}\right)}\right) \\
& \triangleq \bar{L}_{j}(t)
\end{aligned}
$$

Assume $L_{j}(t)>0$ (In Lemma 4.6, we prove that if controller gain $K_{L_{j}}(k)$ is selected sufficiently large, $L_{j}(t)>d>0$ holds for all $t \geq 0$ ), and because

$$
\frac{d \bar{L}_{j}}{d t}=\left[K_{L_{j}}(k) L_{d_{j}}+W_{j-1}\left(\left|\tilde{\alpha}_{j}(k)\right|\right)-K_{L_{j}}(k) L_{j}(k)\right] e^{-K_{L_{j}}(k)\left(t-\tau_{k}\right)}
$$

$\frac{d \bar{L}_{j}}{d t} \geq 0$ or $\frac{d \bar{L}_{j}}{d t}<0$ over interval $\left[\tau_{k}, \tau_{k+1}\right)$. In other words, $\bar{L}_{j}(t)$ is a monotone function over $\left[\tau_{k}, \tau_{k+1}\right)$. Thus $\sup _{\tau_{k} \leq t<\tau_{k+1}} L_{j}(t)$ is obtained when $t=\tau_{k}$ or $t \rightarrow \tau_{k+1}$, i.e.

$$
\begin{aligned}
L_{j}(t) & \leq \sup _{\tau_{k} \leq t<\tau_{k+1}} L_{j}(t) \\
& =\max \left\{\bar{L}_{j}\left(\tau_{k}\right), \bar{L}_{j}\left(\tau_{k+1}\right)\right\} \triangleq M_{L_{j}}(k)
\end{aligned}
$$

Note that $\left|\tilde{L}_{j}(t)\right|=\left|L_{d_{j}}-L_{j}(t)\right| \leq L_{j}(t)+L_{d_{j}}$, thus

$$
\left|\tilde{L}_{j}(t)\right| \leq L_{d_{j}}+M_{L_{j}}(k)
$$

By inequalities (26-27), (25) can be further bounded

$$
\left|\left[\begin{array}{c}
v_{j}(t) \\
\omega_{j}(t)
\end{array}\right]\right|_{\infty} \leq\left(1+M_{L_{j}}(k)\right) \max \left\{K_{L_{j}}(k)\left(L_{d_{j}}+M_{L_{j}}(k)\right), K_{\alpha_{j}}(k)\left|\tilde{\alpha}_{j}(t)\right|\right\}
$$

with $\tilde{\alpha}_{j}(t)=\alpha_{d_{j}}-\hat{\alpha}_{j}(t)$ satisfying

$$
\dot{\tilde{\alpha}}_{j}=-K_{\alpha_{j}}(k) \tilde{\alpha}_{j}, t \in\left[\tau_{k}, \tau_{k+1}\right)
$$

with initial value $\tilde{\alpha}_{j}\left(\tau_{k}\right)$. From the solution of the above ODE, it is obvious that $\left|\tilde{\alpha}_{j}(t)\right|<\left|\tilde{\alpha}_{j}\left(\tau_{k}\right)\right|$, then it is straightforward to show that if the condition (10) is satisfied, the inequality (11) holds. 